\title{
Geometric Feature Enhancement via Iterative High-Boost Filtering
}

\author{
Cao Juming ${ }^{1,2}$ and Wushour Slamu ${ }^{1}$ \\ ${ }^{1}$ School of Electronic and Information Engineering, Xi' an Jiaotong University \\ Xi'an, Shaanxi, 710049, P.R. China \\ ${ }^{2}$ Department of Compter Science, Baoji University of Arts and Sciences, Baoji, \\ Shaanxi, 721000, P.R. China \\ caojuming@stu.xjtu.edu.cn,Wushour@xju.edu.cn
}

\begin{abstract}
We propose a new technique for enhancing high frequency geometric features and geometric texture for scatter point clouds. We generalize the classical High-Boost filtering of signal and image processing to the geometric feature manipulation for $3 D$ point clouds. We get a smoothed version of the input point clouds by Adaptive Moving Least Squares (MLS) based point clouds smoothing operator. The distance fields between the point clouds and their corresponding smoothed version are regarded as the high frequency geometric features and geometric textures intuitively. High Boost operations are performed by iteratively updating the position of the input points along the normal direction which is proportion to the distance between the point clouds and their corresponding smoothed versions with given geometric enhancement scale factor. The effectiveness of the proposed method is demonstrated by several examples with both synthetic and real scanned point clouds.
\end{abstract}

Keywords: Point Clouds, Moving Least Squares, Feature Enhancement, High Boost Filtering

\section{Introduction}

Acquiring 3D models from real-world objects by 3D digital photography and scanning systems $[1,2]$ is a relatively inexpensive process for an increasing number of applications. For example, it can help 3D designer to create detailed models at high resolution, which in turn improves virtual realism in entertainments and film productions. It is also helpful in rapid prototyping, reverse engineering, and capture of cultural artifacts. The acquired data is generally a dense set of points, where each point samples a 3D location and possible additional attributes such as normal information and material properties.

Various researchers proposed the point sample as simple and versatile graphics primitives complementing conventional geometric elements, such as triangles or splines. Most efforts have been devoted to point clouds acquisition, processing ,rendering and point based modeling [2]. As opposed to the traditional polygonal mesh, such a purely point based representation is particularly useful when dealing with large and complex data set, since there is no connectivity information to worry about during the modeling. There are many methods for defining and approximating point set surfaces. Moving Least Squares (MLS) based surfaces has developed into one of the state-of-art methods [3].

High frequency geometric contributes to the geometric detail, while low frequency signals account for the overall geometric shape. The high frequency information of the underlying model plays an essential role in its visual appearance. The term features has different meanings depending on the discipline and application. In geometrical modeling and computer 
graphics the term geometric features is used for free form features of 3D shapes. A free form feature is a visually prominent characteristic of shape, including salient edges, ridges and valley lines as well as geometric textures such as thorns, scales, and bark.

As normal and connectivity are not provided in the point clouds, the estimation of reliable normal and other geometric properties are non-trivial tasks. The noise and outliers points inherent in point clouds present a second major challenge to geometric feature enhancement. Thirdly, geometric features and geometric textures consist of high frequencies which cannot properly sampled by the finite resolution of the scanning devices. When the underlying surfaces contains high frequency geometric feature and geometric textures , the requirement of being resilient to noise and amplifying the high frequency geometric feature simultaneously is especially challenging since the distinction between the noise and high frequency geometric feature is ambiguous.

Inspired by conceptions of multiresolution point clouds representation[4] and High-Boost Filtering in fields of 1D signal processing and 2D digital image processing[5], a novel high frequency geometric feature enhancement method is proposed in this paper. We take as input an unorganized point cloud. Then we get a smoothed version of the original point cloud using the MLS based point cloud smoothing proposed in literature[6] with given scale factor. Thus the distance fields between the original points cloud and its smoothed version is considered as the high frequency geometry features that need to be enhanced intentionally. The high frequency geometry features are amplified by an iterative process: iteratively updating the position of the input points along the normal direction which is proportion to the distance between the point clouds and their corresponding smoothed versions with given geometric enhancement scale factor (Figure 1).

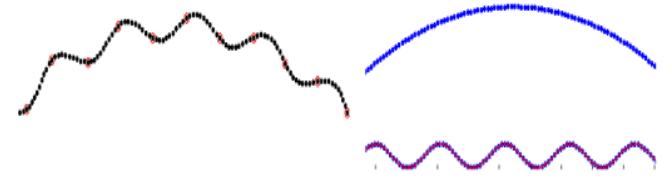

(a) (b)

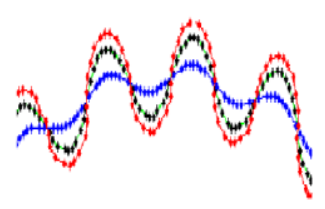

(c)

Figure 1. Principles of our High Frequency Geometric Feature Enhancement Method. (a) Original Point Cloud, (b)Low Frequency Component(top) and High Frequency Geometric Features(Bottom), (c)One Time(Blue), Two Times(Black) and Three Tomes(Red)Iterative High Boost Geometric Feature Enhancement

The advantage of our approach is following: Our method is opening and scalable, some state of the art progress in the fields of point clouds normal estimation, point clouds smoothing can be integrated in our framework conveniently., the input data consists of only unorganized points, neither global nor local parameterization is needed priory. The presented method needs no surface reconstruction and easy to be implemented as well as its lower computational complexity.

The paper is organized as following: After discussing the related works in Section 2, we give a brief introduction to the concept of high boost filtering in Background of digital image processing in Section 3. We introduce the MLS based point cloud smoothing methods and normal estimation in Section 4. In Section 5, we discuss the method of iteratively updating the position for high frequency geometric feature manipulation. Section 6 concern the implementation and result of our method. We conclude the paper with final remarks and future focus of works in Section 7. 


\section{Related Works}

Signal processing as an approach to surface fairing in the irregular setting was first considered by Taubin[7]. He defines frequencies as the eigenvectors of a discrete Laplacian generalized to irregular triangulations. Guskov et al., [8] apply similar algorithm to the subdivision mesh based on wavelet theories, They generalize the Taubin's[7] method to the no uniform setting. Tasdizen et al., [9] applied high-boost filter to face normal on a surface represented as a level set of volumes. After the filtering, a level set method that is computationally expensive is used for manipulating the surface in order to fit it into the processed normal. Yagou et al., [10] Yagou et al., developed a 3D Shape enhancement method based on the high-boost filter to face normal on a triangle mesh and updating mesh vertex positions to make them adapt to the boosted normal. However, this method will introduce the aliasing. Recently Ji et al., [11] Present method for enhancing shape features of a mesh surface by moving mesh vertices from low-curvature regions to high-curvature regions or feature regions. The movement of the vertices, also called vertex flow, is driven by minimizing an objective function defined to take into account several important considerations in mesh improvement.

Aforementioned schemes are applicable to polygonal meshes or triangular meshes only, relying heavily on globally consistent connectivity information between sampled vertices. In contrast, in this paper, our novel detail-preserving editing framework is purely point-based without reconstructing the triangular meshes, which makes it particularly convenient for the large models obtained by scanning devices.

Considerable research has been devoted to the efficient modeling, shape editing and deformation of point-sampled geometry. By transferring several 2D image editing techniques to irregular 3D point setting, Zwicker et al., [12]presented a system called Pointshop 3D for interactive shape and appearance editing of point-sampled surfaces. The system is supported by a powerful point cloud parameterization and a dynamic re-sampling scheme. Pauly et al., [4] Designed a powerful and versatile hierarchical representation for point-based Modeling based on the multi-resolution techniques.

L. Miao, et al., [6] Present a method to compute a set of variation modes for point set surfaces, and represent the point set surfaces as a linear combination of the variation modes, yielding a generative representation for point set surfaces. Then we use this representation to synthesize new shapes for denoising, smoothing and detail enhancement.

Y. Miao et al., [13] generalize the ${ }^{l m}$ spectral field filtering method propose in [7] to the point sampled surface fields and utilize the high order transfer function to the construction of band pass geometric filtering. They utilize the above techniques to the task of geometric detail manipulation such as detail-preserving editing framework, geometric detail scaling and enhancement and so on.

Y. Liu et al., [14] propose an algorithm to build a set of orthogonal Point-Based Manifold Harmonic Bases (PB-MHB) for spectral analysis over point-sampled manifold surfaces. They define a set of orthogonal bases over the point cloud for further spectral geometric analysis and processing tasks such as salient feature manipulation, geometric filtering and so on.

The aforementioned point based geometric feature manipulation method almost all based on the Laplacian eigenbasis on the point clouds which requires finding a partial spectral decomposition of a large symmetric matrix. This computation is too expensive to be applied in practice to anything but small point clouds. The method that we present here is based on the theory of high boost filtering [5] and multiresolution point cloud representation techniques [4]which avoids explicit computation of the underlying basis. The core operators of our method are point clouds smoothing and iterative updating the points along normal direction. 
Our method straightforward and easy to implemented. To my knowledge, fewer works directly address the problem concern geometric feature enhancement for point clouds.

\section{High Boost Filtering}

The high boost filtering technique is commonly used in the signal processing and digital image processing [5]. It is often desirable to enhance high frequency component while still keeping the low frequency components. The high boost filter is a simple sharpening operator via a procedure which subtracts an unsharp, or smoothed, version of an image from the original image. The complete high boost process is shown in the Figure 2. The process for high boost in digital image is also presented in Eqn.(1) Eqn.(2) respectively, where $k$ is a scaling constant. Reasonable values for $k$ vary between 0.2 and 0.7 , with the larger values providing increasing amounts of sharpening.

$$
\begin{aligned}
& g(x, y)=f(x, y)-f_{\text {smooth }}(x, y) \\
& f_{\text {sharp }}(x, y)=f(x, y)+k g(x, y)
\end{aligned}
$$

The high boost filtering can be implemented using an appropriately defined low-pass filter to produce the smoothed version of an image, which is then pixel subtracted from the original image in order to produce a description of image edges, i.e., a high-passed image.

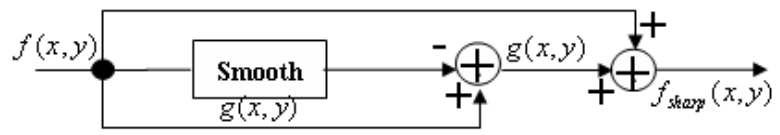

\section{Figure 2. The Flowchart of High Boost Filtering}

\section{Modified MLS based Point Cloud Smoothing and Normal Estimation}

Let us define point cloud as a simple set of 3D point coordinates $\mathfrak{P}=\left\{p_{1}, p_{2}, \ldots p_{n}\right\}, p_{i} \in R^{8}, i=1,2, \ldots, N$ without any normal or connectivity information. The data points are unstructured and suppose to belong to a 2-manifold surface. Let $N=|\mathfrak{P}|$ be the cardinal of the point set. Let $\mathfrak{N}_{p}=\left\{p_{i}\left|p_{i} \in \mathfrak{P},\right| p_{i}-p \mid<\delta\right\}$ be the $\delta$ neighborhoods of poin t $p_{i} \in \mathfrak{P}$ with radius $\delta$. We choose a KD-tree implementation to accelerating the query operation on point clouds such as the finding ${ }^{\mathfrak{N}_{p}}$ of every ${ }^{p \in \mathfrak{P}}$. The KD-tree can be constructed only once and can be used in the follows pipeline.

\subsection{Normal Estimation}

Many research works concentrate on the normal estimation and normal direction consistency [15-17]. Most of those methods achieve the accuracy at the price of heavy computational overhead. Here we use the traditional local PCA based method to estimate local normal. For every $p \in \mathfrak{P}^{3}$ and its $\mathfrak{N}_{p}$, let,

$$
\bar{p}=\frac{1}{b} \sum_{p_{i}=\tau_{i}} p_{i}
$$




$$
M=\frac{1}{k} \sum_{\hat{A} \in \mathfrak{T}_{,}}\left(p_{i}-\bar{p}\right)\left(p_{i}-\bar{p}\right)^{t}
$$

Then we get the eigenvalue $\lambda_{1} \geq \lambda_{2} \geq \lambda_{3} \geq 0$ and their corresponding eigenvector $\mathbf{v}_{1}, \mathbf{v}_{2}, \mathbf{v}_{3}$ of matrix $M$. As a result, we define the direction of vector $\mathbf{v}_{3}$ as the normal estimation. Weuse the method proposed in [18] for locally orientation consistency and normal field smoothing.

\subsection{Modified MLS based Point Cloud Smoothing}

We use a point cloud smoothing method based on Modified MLS surface approximation proposed by Y. L.Miao, et al., [6]. The traditional MLS surface[3] of point set $\mathrm{P}$ is defined as the stationary set of a projection operator which takes a point $\mathrm{x}$ nearby the point set onto a polynomial that locally approximates the underlying surface in the vicinity of $x$. MLS surfaces have been widely used for point based modeling and rendering in the last few years. Briefly, this MLS projection procedure at point $\mathrm{x}$ takes two steps.

First, a reference plane $H$ is fitted by minimizing the following weighted least squares:

$$
\sum_{i \in v}\left(p_{i} n-D\right)^{2} \theta\left(x, p_{i}\right)
$$

Where $\mathrm{n}$ is the normal vector of the reference plane $H, D$ is the distance of the origin to the reference plane $H$, and $\theta\left(x, p_{i}\right)$ is a positive weighting function. The reference plane $H$ provides local parameterizations of the sample points. Let $q$ be the projection of $x$ on $H$, $\left(u_{i}, V_{i}\right)$ be the local parameterization of $p_{i}$ 's projection on $H$, and $f_{i}=n\left(p_{i}-q\right)$. Then a bivariate polynomial $g(u, V)$ in the reference plane $H$ is fitted by minimizing the following weighted least squares:

$$
\sum_{i \in v}\left(g\left(u_{i}, v_{i}\right)-f_{i}\right)^{2} \theta\left(x, p_{i}\right)
$$

Finally, the projection of $x$ on the polynomial is obtained as the MLS projection:

$$
\psi(x)=q+g(0,0) \mathbf{n}
$$

Based on the MLS projection, Weyrich et al.[19] Implemented a smoothing method, called MLS smoother, by shifting each point $p_{i} \in \mathfrak{P}$ toward $\psi^{2}(x)$ by some distance:

$$
(1-\alpha) p_{i}+\alpha \psi\left(p_{i}\right) \rightarrow p_{i}
$$

Where $\alpha \in[0,1]$. The weighting function in the above the MLS projection is given by a Gaussian $\dot{\theta}\left(x, p_{i}\right)=e^{-\left|-p_{1}\right|^{2} /\left(2 h^{2}\right)}$, where $h$ is an user-specified constant. Generally, a larger $h$ will give a smoother surface.

The Modified MLS based point cloud smoother incorporates the idea of the bilateral denoising methods [20-22] by introducing a new weight function ${ }^{w\left(x, p_{i}\right)}$ that is formulated as:

$$
w\left(x, p_{i}\right)=\theta\left(x, p_{i}\right) \phi\left(x, p_{i}\right)
$$


Where $\phi\left(x, p_{i}\right)$ is defined as:

$$
\phi\left(x, p_{i}\right)=\exp \left(-\frac{\left\|x-\prod_{p}(x)\right\|^{2}}{2 s^{2}}\right)
$$

Let $\mathrm{n}_{i}$ be the normal at point ${ }^{p_{i}}$, as shown in Figure 3, the linear prediction $\prod_{p_{i}}(x)$ for $x$ is defined as its projection on the tangent plane of $p_{i}$, then

$$
\prod_{p_{i}}(x)=x+\left(\left(p_{i}-x\right) \cdot \mathbf{n}_{i}\right) \cdot \mathbf{n}_{i}
$$

Free parameters $s_{s}$ and $h$ should be tuned manually as be proposed in literature[6]. Figure 3 illustrates the relationship between $\prod_{p_{i}}(x), \mathrm{n}_{i}$ and $p_{i}$.

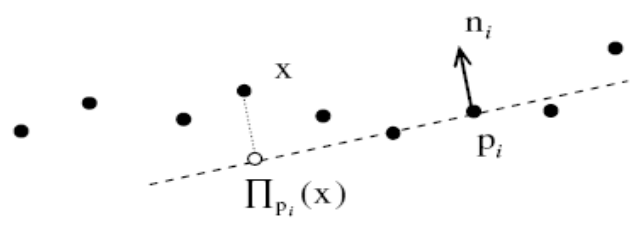

Figure 3. Linear Prediction for Point $x$ by the Information of a Nearby Point $p_{i}$

\section{Iterative Position Updating for Shape Enhancement}

Our high boost filtering is an iterative process, that is: we iteratively update the position of the original point along the normal direction. The distance between the original point and its updated position is proportion to the given shape enhancement factor $\lambda$. For every point

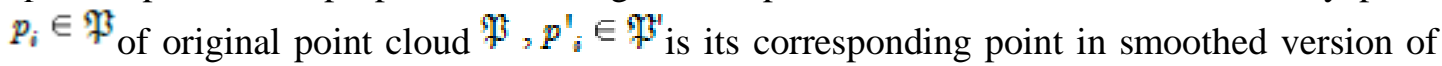
$\mathfrak{P}$ where the smoothed version of the original point cloud is denoted by $\mathfrak{P}$. Let $p_{i}{ }^{\mu} \in \mathfrak{P}^{\mu}$ denote the position of updated position of $p_{i} \in \mathfrak{P}^{\mathrm{U}}$, where $\mathfrak{P}^{\mu}$ means the point cloud after iterative high boost filtering.

For each iteration, we update the position of every point $p_{i} \in \mathfrak{P}$ as following: we moving the position of $p_{i}$ along normal direction of $p_{i}$, and the distance between the point $p_{i}$ and its updated position $p_{i}{ }^{u}$ is proportion to the given feature enhancement factor $\lambda$. Let $\mathrm{n}_{i}$ be normal of point $p_{i}$ estimated using method aforementioned in Chapter 4.3. The position updating method is formulated as:

$$
p_{i}{ }^{u}=p_{i}+\lambda \cdot \mathbf{n}_{i} \cdot\left(\left(p_{i}^{\prime}-p_{i}\right) \cdot \mathbf{n}_{i}\right) /\left\|\mathbf{n}_{i}\right\|^{2}
$$

Figure 4, illustrate method of our position updating in once iteration. For $p_{i}$ (denotes as letter ' $C^{\prime}$ ) and its corresponding point in smoothed version $p_{{ }_{i}}^{\prime}$ (denotes as letter ' $\left.\mathrm{A}^{\prime}\right) . \mathrm{n}_{i}$ is on the vector $B D, C D$ is the projection of vector $p_{i}^{\prime}-p_{i}$ along direction of normal $\mathrm{n}_{i}$, and $\lambda=\|\overrightarrow{C F}\| /\|\overrightarrow{C D}\|$, then position of point ' $\mathrm{F}$ ' is the updated position of point $p_{i}$. The free 
parameter 1 can be tuned manually, in our experiment,we use the value between among $0.1 \sim 0.3$.

The whole iteration processing can be described in pseudo code in Figure 5. In our implement the iteration number $I$ should be tuned manually, the typical $I$ is within the range of 3 12. Too large $I$ could produce geometric feature exaggerated visual effect and need upsample operation to compensate aliasing effect caused by high boost operation.

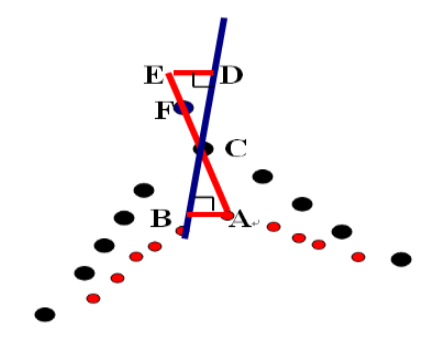

Figure 4. Illustrate Position Updating in Once Iteration

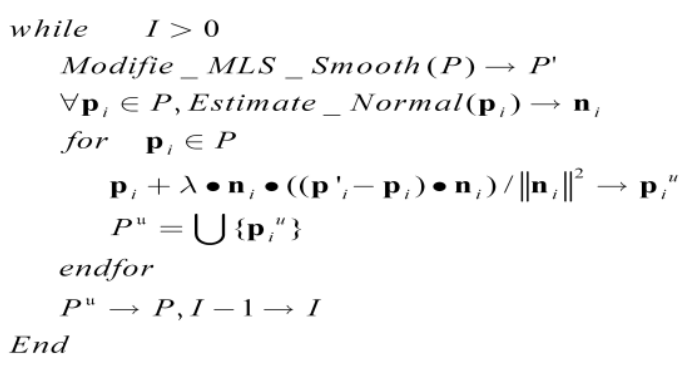

Figure 5. Process of Iterative Position Update

\section{Implementation and Results}

All the algorithms presented in this paper are implemented and tested on a PC with a Pentium 4 Dual Core 2.0GHz CPU, 2GB memory and Windows XP in Matlab environment. Table 1 shows the data statistical, Timing for once iterative position update and tuning of free parameters of Modified MLS smoothing. Table 1 shows the Statistics, Timings and Parameters of High Boost Operation on Different Point Cloud. (s Means second, time units. $R$ is the radius size of the model's bounding sphere).

Table 1. Statistics, Timings and Parameters of High Boost Operation on Different Point Clouds

\begin{tabular}{|c|c|c|c|c|c|}
\hline Point Cloud & Vertices & Iteration & Factor $l$ & $s$ & $h$ \\
\hline Bunny & 34834 & $29.0634 \mathrm{~s}$ & 0.186 & $0.0042^{R}$ & $0.043^{R}$ \\
\hline Max-Plank & 52809 & $43.1206 \mathrm{~s}$ & 0.207 & $0.0068^{R}$ & $0.038^{R}$ \\
\hline Sheep & 159331 & $168 \mathrm{~s}$ & 0.165 & $0.0042^{R}$ & $0.035^{R}$ \\
\hline
\end{tabular}

Figure 6 shows results of our high frequency feature enhancement approach applied on the Bunny Model. For original point cloud with 34834 vertices, we iteratively updating the 
position of the original points along the normal direction thus we get the high frequency geometric feature enhancement effective. Original point clouds Figure 6. (a)) and the smoothed version (Figure 6. (b)) are rendered with smooth shading. For given feature enhancement factor $\lambda=0.186$, we iteratively updating the position of point along the direction of normal thus we get the result of geometric feature enhancement respectively. Figure 6 $(b, c, d, e, f)$ shows the result of different iteration number $I: \quad I=1, I=3, I=5$, $\mathrm{I}=9, \mathrm{I}=12, \mathrm{I}=5$, respectively.
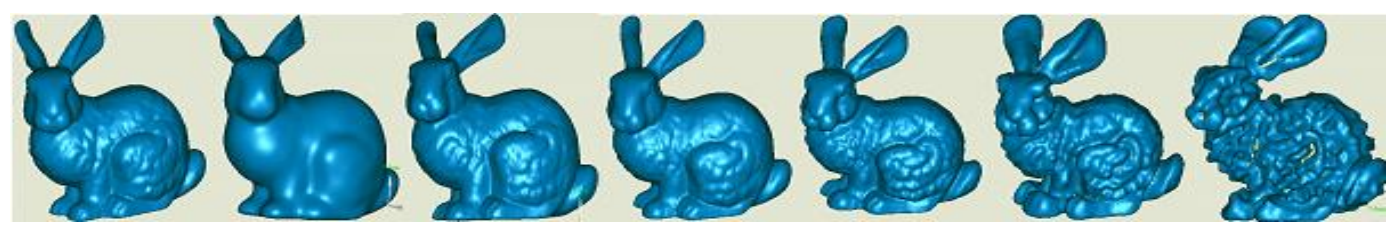

\section{Figure 6. High Boost Geometric Feature Enhancement on the Point Cloud Bunny}

Figure 7 shows results of our high frequency feature enhancement approach applied on the Max_Planck with 52809 vertices. For given feature enhancement factor $\lambda=0.206$, we iteratively updating the position of point along the direction of normal thus we get the result of geometric feature enhancement point cloud models respectively. Original point clouds(Figure 7 (a)) and the smoothed version (Figure 7 (b)) are rendered with smooth shading Figure 7 (b,c,d,e,f) shows the result of different iteration numbers $I$ : $I=1, I=3, I=5$, $\mathrm{I}=9, \mathrm{I}=12, \mathrm{I}=5$, respectively.

Figure 8 shows results of our high frequency feature enhancement approach applied on the Max_Planck with 52809 vertices. Figure 9 shows results of our high frequency feature enhancement approach applied on the Moai from aim@shape.net with 19862 vertices. For given feature enhancement factor we iteratively updating the position of point along the direction of normal thus we get the result of geometric feature enhancement respectively. Figure $7(\mathrm{c}, \mathrm{d}, \mathrm{e}, \mathrm{f})$ shows the result of different iteration number I: I=2,3,12,13 respectively.

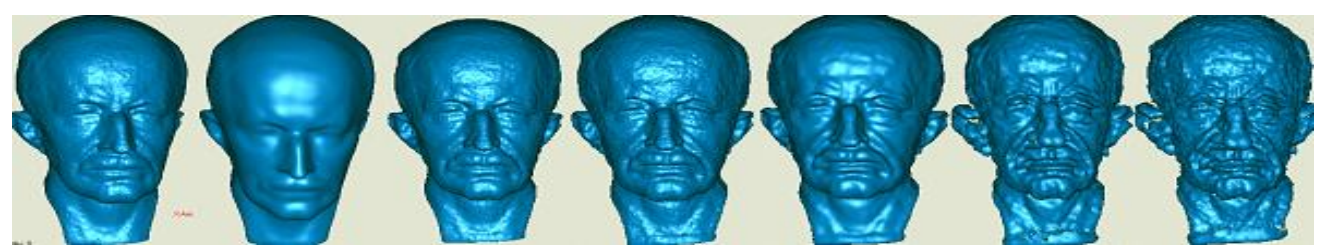

Figure 7. High Boost Geometric Feature Enhancement on the Point Cloud MaxPlanck

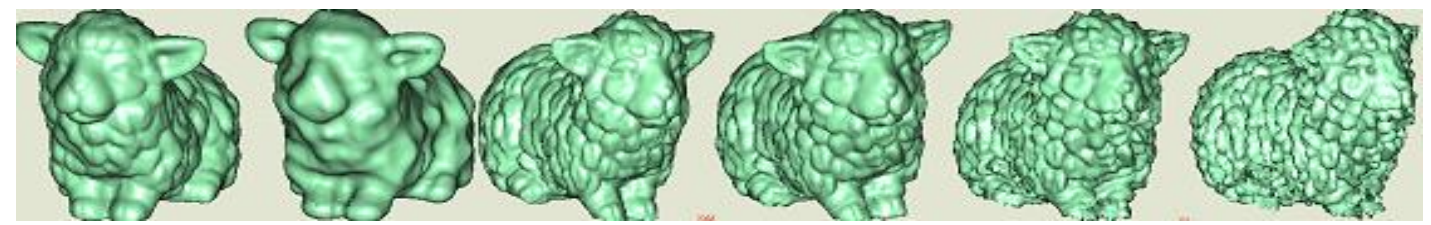

Figure 8. High Boost Geometric Feature Enhancement on the Point Cloud Sheep. Original Point Clouds (a) and its Smoothed Version (b), Rendered with Smooth Shading 


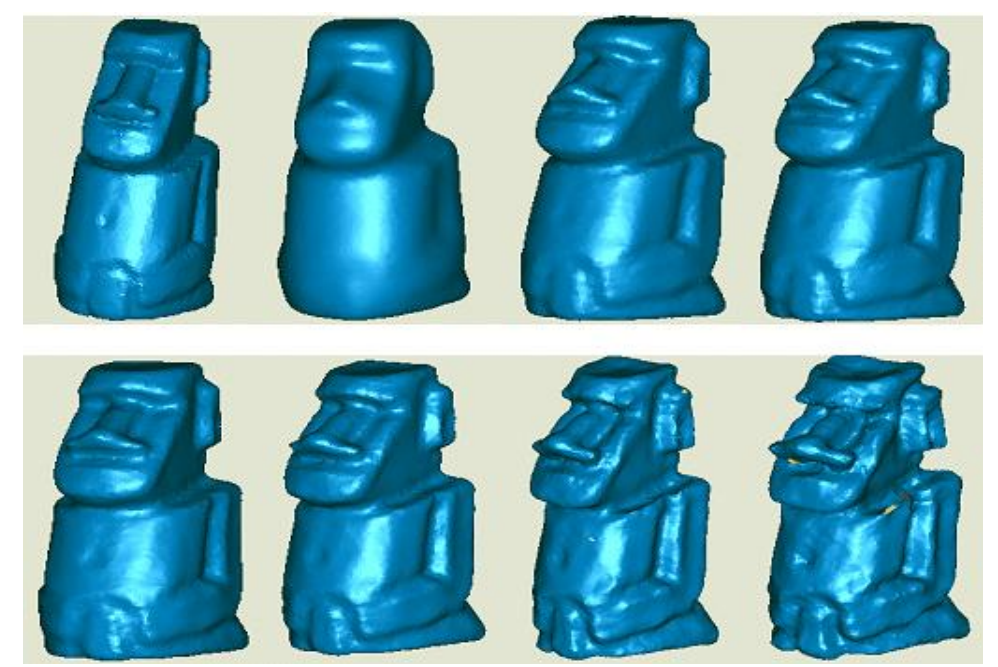

\section{Figure 9. High Boost Geometric Feature Enhancement on the Point Cloud Moai from aim@shape.net with 19862 Vertices}

It is intuitively: for given feature enhancement factor $\lambda$ and iteration number $\mathrm{I}$, too small iteration number I cannot produce obvious visual geometric feature enhancement effective (Figure 6 a, Figure 7 a, and Figure 8 a respectively). Too big iteration number I may produce the exaggerate feature enhancement effective (Figure 6 e,f, Figure 7 e,f and Figure 8 e,f respectively). Our experimental results (Figure 6 c, d, Figure 6. c,d, Figure 7 c, d respectively) show that our geometric feature enhancement approach can always yield natural deformation when the accumulated enhancement factor $\lambda_{2}=\lambda \cdot I$ (which is define as the product of enhancement Factor $\lambda$ and iteration number I) is with the range of $0.4 \leq \lambda_{\Sigma} \leq 1.1$. How to tuning those free parameters automatically and geometrical adaptively are the focus of our future works.

\section{Conclusions and Future Work}

We generalize the 2D high boost filtering to the fields of 3D geometric feature enhancement. The high boost geometric feature is enhanced via iterative updating positions of every point along normal direction. The distance between the original points and updated position is proportion to the distance between the original point and its corresponding point in soothed version.

Our method is simple and straightforward. It is easy to be implemented. Input of our method consists of a set of unorganized points; neither global nor local parameterization is needed priory. There is no need of ROI (region of interesting) being specified annually. Only few free parameters with intuitive geometric significance should be tuning manually.

Yet there is still room for improving performance of our high boost geometric enhancement method. Although we can tune the free parameters of our method manually, the relationship both quantity and quality between those free parameters is not known. Adopt the strategies proposed in literature[23] and tuning those free parameters automatically and geometrically is focus of our further works. Our experiment shows that to larger accumulated enhancement factor $\lambda_{\Sigma}$ may cause obviously geometric feature exaggerate as well as geometric aliasing. How to eliminate those geometric aliasing is also focus of our future research works. 


\section{Acknowledgements}

This work is supported by the National Natural Science Foundation of China under Grant NO: 61063024.

\section{References}

[1] F. Blais, "Review of 20 years of range sensor development", Journal of Electronic Imaging, vol. 13, no. 1, (2004).

[2] M. Gross and H. Pfister, "Point-Based Graphics, Morgan Kaufmann Pub, (2007).

[3] Z.-Q. Cheng, Y.-Z. Wang, B. Li, K. Xu, G. Dang and S.-Y. Jin, "A Survey of Methods for Moving Least Squares Surfaces", Proceedings of Volume Graphics, (2008).

[4] M. Pauly, L. Kobbelt and M. Gross, "Multiresolution Modeling of Point-Sampled Geometry", Eidgenössische Technische Hochschule Zürich, (2002).

[5] R. C. González and R. E. Woods, "Digital image processing”, Prentice Hall, (1993).

[6] L. Miao, J. Huang, X. Liu, H. Bao, Q. Peng and B. Guo, "Computing Variation Modes for Point Set Surfaces", Eurographics Symposium on Point-Based Graphics, (2005).

[7] G. Taubin, "A Signal Processing Approach to Fair Surface Design", Proceedings of the 22nd annual conference on Computer graphics and interactive techniques, (1995).

[8] I. Guskov, W. Sweldens and P. Schroder, "Multiresolution Signal Processing for Meshes", Proceedings of the 26th annual conference on Computer graphics and interactive techniques, (1995).

[9] T. Tasdizen, R. T. Whitaker and P. B. a. S. Osher, "Geometric Surface Processing via Normal Maps", ACM Transactions on Graphics, vol. 22, no. 4, (2003).

[10] H. Yagou, A. Belyaev and D. Wei, "High-Boost Mesh Filtering for 3-D Shape Enhancement", Journal of Three Dimensional Images, (2003).

[11] Z. Ji, L. Liu and B. Wang, "Feature Enhancement by Vertex Flow for 3D Shapes", Computer Aided Design \& Applications, (2011).

[12] Z. Matthias, M. Pauly and O. Knoll, "Pointshop 3D: An interactive system for point-based surface editing", ACM Trans. Graph, vol. 21, no. 3, (2002).

[13] Y. Miao, J. Feng and C. Xiao, "High frequency geometric detail manipulation and editing for point-sampled surfaces", Visual Comput., (2007).

[14] Y. Liu, B. Prabhakaran and X. Guo, "Point-Based Manifold Harmonics", Transactions on Visualization and Computer Graphics, (2011).

[15] B. Li, R. Schnabel and R. Klein, "Robust normal estimation for point clouds with sharp features", Computers \&Graphics, vol. 106, no. 34, (2010).

[16] K. Klasing, D. Althoff and D. Wollherr, "Comparison of Surface Normal Estimation Methods for Range Sensing Applications”, Robotics and Automation, 2009, ICRA'09, IEEE International Conference, (2009).

[17] T. K. Dey, G. Li, and J. Sun, "Normal Estimation for Point Clouds: A Comparison Study for a Voronoi Based Method", Eurographics Symposium on Point-Based Graphics, (2005).

[18] J. Cao, S. Wushour and X. Yao, "Robust sharp features infer from point clouds", SPIE Optical Metrology, (2011).

[19] T. Weyrich, M. Pauly and R. Keiser, "Post-processing of Scanned 3D Surface Data", Eurographics Symposium on Point-Based Graphics, (2004).

[20] L. K. Wah, "Mesh Denoising and Feature Extraction from Point Cloud Data", The University of Hong Kong, (2009).

[21] K.-W. Lee and W.-P. Wang, "Feature-Preserving Mesh Denoising via Bilateral Normal Filtering", Ninth International Conference on Computer Aided Design and Computer Graphics, (2005).

[22] C. C. L. Wang, "Bilateral Recovering of Sharp Edges on Feature-insensitive Sampled Meshes", Visualization and Computer Graphics, IEEE Transactions, vol. 12, no. 4, (2006).

[23] B. Kim, and J. Rossignac, "GeoFilter: Geometric Selection of Mesh Filter Parameters", EUROGRAHICS, (2005). 


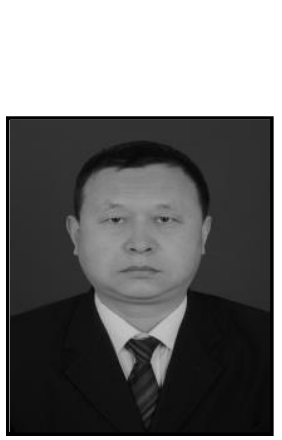

\section{Authors}

Cao Juming, he is a $\mathrm{PhD}$ candidate at the school of Electronic and Information Engineering, Xi' an Jiaotong University, Xi'an, P.R. China. $\mathrm{He}$ received his BSc in mathematic from Baoji University of Arts and Sciences, Baoji, P.R. China in 1992 and MSc in computer science and engineer from XiDian university, Xi' an, P.R. China in 2004. His research interests lies in the area of Computer Graphics, Geometric modeling, Digital image processing, Virtual and argument reality.

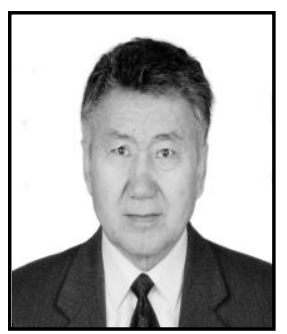

Wushour Slam, he graduated from the Physics Department of Xinjiang University, Urumchi, P.R. China. He is now a supervisor of doctoral postgraduates in computer science and engineer at Electronic and Information Engineering, Xi' an Jiaotong University, Xi'an, P.R. China. His research interests lies in the area of Computational linguistics, Computer Graphics. 
International Journal of Signal Processing, Image Processing and Pattern Recognition Vol.7, No.2 (2014) 